\title{
Kommentar zu „IKT-Anbieter als Thema der Wirtschaftsinformatik?"
}

\section{DOI 10.1007/s11576-013-0356-4}

\section{Der Autor}

Prof. Dr. Martin Kütz ( $\varangle)$

Lehrgebiet Wirtschaftsinformatik

Hochschule Anhalt

Lohmannstr. 23

06366 Köthen (Anhalt)

Deutschland

m.kuetz@inf.hs-anhalt.de

Online publiziert: 2013-02-22

This article is also available in English via http://www.springerlink.com and http://www.bise-journal.org: Kütz M (2013) Comments on "ICT Providers: A Relevant Topic for Business and Information Systems Engineering?". Bus Inf Syst Eng. doi: 10.1007/ s12599-013-0256-6.

(C) Springer Fachmedien Wiesbaden 2013

„IKT-Anbieter als Thema der Wirtschaftsinformatik?" (Hess et al. 2012) - Was für eine Frage! Ist die Erstellung von IT-Leistungen etwa kein Thema der Wirtschaftsinformatik? Wenn wir uns mit der Unterstützung (betriebs-) wirtschaftlicher Aktivitäten durch informationsverarbeitende und kommunikationstechnische Systeme befassen, dann müssen wir uns doch notgedrungen nicht nur mit dem Aspekt des ITDemand, sondern zwangsläufig auch mit den Aspekten des IT-Supply und der IT-Governance befassen. Wer Ressourcen verbraucht, steht immer vor der Frage des „Make or Buy“ und er darf sich im Fal- le einer „Buy“-Entscheidung nicht darauf beschränken, die bezogene Leistung als Blackbox zu betrachten. Wer optimieren will, muss die gesamte SupplyChain offenlegen und einbeziehen. Insofern sind natürlich auch die externen und nicht nur die internen IKTProduzenten ein elementares Thema der Wirtschaftsinformatik.

Aber was wäre, wenn man die Frage anders beantwortet? Wenn man den adressierten CIO einmal nicht als den obersten IT'ler, sondern tatsächlich als obersten Informationsmanager ansieht, der die Informationswirtschaft einer Unternehmung (oder ähnlicher Organisationen) führt? Der sich mit Informationen befasst und nicht mit Techniken und Systemen? Der tatsächlich die Informationsversorgungsprozesse einer Organisation gestaltet und nicht nur (technische) Infrastrukturen bereitstellt? Was bliebe, wäre ein (durchaus faszinierendes) Gemisch aus Betriebsorganisation und Informationsmanagement. Die Informatik wäre aber dann nur noch Vehikel und nicht mehr Kernthema. Zwar wäre es durchaus zu wünschen, wenn wir in der betrieblichen Praxis wieder mehr über Organisation und Information sprechen würden und nicht immer sofort über Plattformen und Systeme. Wenn wir uns auf Probleme einlassen und auch einmal (kreativ) nach nicht-technischen Lösungen suchen würden und wenn wir nicht immer nur versuchen würden, alle Fragestellungen mit massivem IT-Einsatz auf die Seite zu räumen. Aber würden wir dann noch über Wirtschaftsinformatik sprechen, wäre sie als eigenständige Disziplin sinnvoll oder gar notwendig? Wohl eher nicht.
Warum die Wirtschaftsinformatik sich zwangsläufig mit der Produktion von IKT-Leistungen und daher auch mit den Produzenten dieser Leistungen befassen muss, ergibt sich auch daraus, dass es ja nicht nur um den Einsatz von IKT im Umfeld wirtschaftlicher Aktivitäten geht, sondern auch um die Wirtschaftlichkeit genau solcher Aktivitäten. Wann macht es Sinn, welche IKT-Systeme zu nutzen? Wobei Sinn nicht zuletzt auch den wirtschaftlichen Sinn bedeutet. Ist der Einsatz wirtschaftlich zu rechtfertigen? Wer befasst sich mit der wirtschaftlichen Seite der IKT, wenn das nicht die Wirtschaftsinformatik tut? Insofern ist sie last, but not least auch eine Betriebswirtschaftslehre des IKT-Bereichs. Es geht eben nicht nur um Verwendungswirtschaftlichkeit, sondern auch um Bereitstellungswirtschaftlichkeit. Und wer den Wertbeitrag der IT (in Organisationen) ermitteln will, muss notgedrungen die Leistungserstellung selber untersuchen. Und dann muss er, ob er will oder nicht, auch die Leistungsersteller ins Visier nehmen.

Dass das Thema offenbar erst jetzt „entdeckt" wird, erstaunt schon. Aber: Besser spät als nie. Es ist gut, wenn wir (endlich) auch diesen Aspekt in unsere Arbeit einbeziehen. Unsere Kollegen in der Praxis werden es begrüßen.

\section{Literatur}

Hess T, Loos P, Buxmann P, Erek K, Frank U, Gallmann J, Gersch M, Zarnekow R, Zencke P (2012) IKT-Anbieter als Thema der Wirtschaftsinformatik? WIRTSCHAFTSINFORMATIK 54(6):355-362 